\title{
An Ostrowski type inequality in two dimensions using the three point rule
}

\author{
G. Hanna P. Cerone J. Roumeliotis*
}

(Received 7 August 2000)

\begin{abstract}
An Ostrowski Type inequality in two dimensions for double integrals on a rectangle is developed. The resulting integral inequalities are valid for the class of functions with bounded first derivatives. They are employed to approximate the double integral by up to 6 one dimensional integrals and nine functions evaluations. Examples using the resulting cubature formulae are presented.
\end{abstract}

* School of Communications and Informatics, Victoria University of Technology, PO Box 14428, MCMC, Victoria 8001, AustraliA. mailto:georgey@matilda.vu.edu .au, mailto:pc@matilda.vu.edu.au, mailto: John. Roumeliotis@vu . edu . au

${ }^{0}$ See http: //anziamj . austms .org. au/V42/CTAC99/Hann for this article and ancillary services, (C) Austral. Mathematical Soc. 2000. Published 27 Nov 2000. 


\section{Contents}

1 Introduction

C672

2 The Ostrowski Inequality

C673

3 The Results

C674

4 Numerical Results

C682

References

C688

\section{Introduction}

Cubature formulae are most often evaluated as iterated one-dimensional integrals. The approach is straightforward but has some disadvantages. Two of which are that the error estimates are unnecessarily large, since they too rely on embedding the one-dimensional error results, and it is often difficult to discretise regions that are other than ideal. That is, regions whose boundaries lie on coordinate lines of some orthogonal system.

In this paper we develop a three point cubature rule for two-dimensional rectangular regions. An a priori error bound is obtained for functions whose first partial derivatives exist and are bounded. The term "three point" is used to draw an analogy with Newton-Cotes type rules where sampling occurs at 
the boundary and interior points. The rule presented here approximates a two-dimensional integral via application of function evaluations and onedimensional integrals at the boundary and interior points. That is, up to nine function points and six one-dimensional integrals. A parametrisation, similar to that of [2], is employed to distinguish rule type. If the one-dimensional integrals are not known, they themselves can be approximated to produce a cubature rule consisting only of sampling points.An additional three point rule, as in [2], may be subsequently used, or indeed any other desired quadrature rule. (For example, the optimal rules at $[4,7]$ ). As a result the error bound will be larger.

The method presented here is based on Ostrowski's integral inequality, and as such is amenable to the production of error bounds for a variety of norms. In addition smoother and product integrands may also be considered as has been done for one-dimensional integrals, see for example $[2,3,6]$.

\section{The Ostrowski Inequality}

The classical Ostrowski integral inequality in one dimension stipulates a bound between a function evaluated at an interior point $x$ and the average of the function of over an interval. That is,

$$
\left|f(x)-\frac{1}{b-a} \int_{a}^{b} f(t) d t\right| \leq\left[\frac{1}{4}+\frac{\left(x-\frac{a+b}{2}\right)^{2}}{(b-a)^{2}}\right](b-a)\left\|f^{\prime}\right\|_{\infty}
$$


for all $x \in[a, b]$, provided that $f^{\prime} \in L_{\infty}(a, b)$ and $f:[a, b] \rightarrow \mathrm{R}$ is a differentiable mapping on $(a, b)$.

Here, the constant $\frac{1}{4}$ is sharp in the sense that it cannot be replaced by a smaller constant. We also make the observation that the smallest bound is obtained at $x=\frac{a+b}{2}$, resulting in the well-known mid-point inequality.

The Ostrowski result was further developed in [2], where Cerone and Dragomir presented a 3-point inequality and showed that the tightest bound is an average of the mid-point and trapezoidal rules. In the paper [1], Barnett and Dragomir developed a two dimensional version of the Ostrowski inequality.

In the current paper we combine the above two results and develop a two dimensional 3-point integral inequality for functions with bounded first derivatives. An application in the numerical integration of a two-dimensional integral is investigated.

\section{The Results}


Theorem 1 Let $f: \mathrm{R}^{2} \rightarrow \mathrm{R}$ be a differentiable mapping on $\left[a_{1}, b_{1}\right] \times\left[a_{2}, b_{2}\right]$ and let $f_{t_{1}, t_{2}}^{\prime \prime}=\frac{\partial^{2} f}{\partial t_{1} \partial t_{2}}$ be bounded on $\left(a_{1}, b_{1}\right) \times\left(a_{2}, b_{2}\right)$. That is,

$$
\left\|f_{t_{1}, t_{2}}^{\prime \prime}\right\|_{\infty}:=\sup _{\left(x_{1}, x_{2}\right) \in\left(a_{1}, b_{1}\right) \times\left(a_{2}, b_{2}\right)}\left|\frac{\partial^{2} f}{\partial t_{1} \partial t_{2}}\right|<\infty .
$$

Furthermore, let $x_{i} \in\left(a_{i}, b_{i}\right)$ and introduce the parametrisation $\alpha_{i}, \beta_{i}$ defined by

$$
\begin{aligned}
\alpha_{i} & =\left(1-\gamma_{i}\right) a_{i}+\gamma_{i} x_{i}, \\
\beta_{i} & =\left(1-\gamma_{i}\right) b_{i}+\gamma_{i} x_{i},
\end{aligned}
$$

where $\gamma_{i} \in[0,1]$, for $i=1,2$. Then the following inequality holds

$$
\begin{array}{r}
\left|\sum_{k=1}^{3} \sum_{j=1}^{3} C_{k 1} C_{j 2} f_{j k}-\sum_{j=1}^{3}\left(C_{j 1} I_{j 2}+C_{j 2} I_{j 1}\right)+\int_{a_{2}}^{b_{2}} \int_{a_{1}}^{b_{1}} f\left(t_{1}, t_{2}\right) d t_{1} d t_{2}\right| \\
\leq \frac{\left\|f_{t_{1}, t_{2}}^{\prime \prime}\right\|_{\infty}}{4}\left(1+\left(2 \gamma_{1}-1\right)^{2}\right)\left[\left(\frac{b_{1}-a_{1}}{2}\right)^{2}+\left(x_{1}-\frac{a_{1}+b_{1}}{2}\right)^{2}\right] \\
\times\left(1+\left(2 \gamma_{2}-1\right)^{2}\right)\left[\left(\frac{b_{2}-a_{2}}{2}\right)^{2}+\left(x_{2}-\frac{a_{2}+b_{2}}{2}\right)^{2}\right],
\end{array}
$$

given that

$$
\left(f_{j k}\right)=\left(\begin{array}{lll}
f\left(a_{1}, a_{2}\right) & f\left(x_{1}, a_{2}\right) & f\left(b_{1}, a_{2}\right) \\
f\left(a_{1}, x_{2}\right) & f\left(x_{1}, x_{2}\right) & f\left(b_{1}, x_{2}\right) \\
f\left(a_{1}, b_{2}\right) & f\left(x_{1}, b_{2}\right) & f\left(b_{1}, b_{2}\right)
\end{array}\right)
$$




$$
\begin{gathered}
\left(C_{j k}\right)=\left(\begin{array}{rr}
\gamma_{1}\left(x_{1}-a_{1}\right) & \gamma_{2}\left(x_{2}-a_{2}\right) \\
\left(1-\gamma_{1}\right)\left(b_{1}-a_{1}\right) & \left(1-\gamma_{2}\right)\left(b_{2}-a_{2}\right) \\
\gamma_{1}\left(b_{1}-x_{1}\right) & \gamma_{2}\left(b_{2}-a_{2}\right)
\end{array}\right), \\
\left(I_{j k}\right)=\left(\begin{array}{cr}
\int_{a_{1}}^{b_{1}} f\left(t_{1}, a_{2}\right) d t_{1} & \int_{a_{2}}^{b_{2}} f\left(a_{1}, t_{2}\right) d t_{2} \\
\int_{a_{1}}^{b_{1}} f\left(t_{1}, x_{2}\right) d t_{1} & \int_{a_{1}}^{b_{1}} f\left(x_{1}, t_{2}\right) d t_{2} \\
\int_{a_{1}}^{b_{1}} f\left(t_{1}, b_{2}\right) d t_{1} & \int_{a_{1}}^{b_{1}} f\left(b_{1}, t_{2}\right) d t_{2}
\end{array}\right) .
\end{gathered}
$$

Proof: Define the kernel

$$
p(x, t)= \begin{cases}t-\alpha, & t \in[a, x], \\ t-\beta, & t \in(x, b],\end{cases}
$$

where, as above, $\alpha=(1-\gamma) a+\gamma x$, and $\beta=(1-\gamma) b+\gamma x$. Using (6) and integrating by parts we obtain, after some simplification, the identity

$$
\begin{aligned}
& \int_{a}^{b} p(x, t) F^{\prime}(t) d t \\
= & (1-\gamma)(b-a) F(x)+\gamma[(x-a) F(a)+(b-x) F(b)]-\int_{a}^{b} F(t) d t .
\end{aligned}
$$

A two dimensional identity can be developed via repeated application of (7). To this end, we define the mapping

$$
p_{i}\left(x_{i}, t_{i}\right)=\left\{\begin{array}{ll}
t_{i}-\alpha_{i}, & a_{i} \leq t_{i} \leq x_{i}, \\
t_{i}-\beta_{i}, & x_{i}<t_{i} \leq b_{i},
\end{array} \quad \text { for } \quad i=1,2 .\right.
$$


Substituting $p_{1}$ for $p$ and $f\left(t_{1}, \cdot\right)$ for $F(t)$ into $(7)$ gives

$$
\begin{array}{r}
\int_{a_{1}}^{b_{1}} p_{1}\left(x_{1}, t_{1}\right) \frac{\partial f}{\partial t_{1}} d t_{1}=\left(1-\gamma_{1}\right)\left(b_{1}-a_{1}\right) f\left(x_{1}, t_{2}\right)+\gamma_{1}\left(x_{1}-a_{1}\right) f\left(a_{1}, t_{2}\right) \\
+\gamma_{1}\left(b_{1}-x_{1}\right) f\left(b_{1}, t_{2}\right)-\int_{a_{1}}^{b_{1}} f\left(t_{1}, t_{2}\right) d t_{1} .
\end{array}
$$

Employing (7) again with $p_{2}$ as the kernel, $F\left(t_{2}\right)=\int_{a_{1}}^{b_{1}} p_{1}\left(x_{1}, t_{1}\right) \frac{\partial f}{\partial t_{1}} d t_{1}$ as the integrand and expanding with (9) produces the desired identity

$$
\begin{gathered}
\int_{a_{2}}^{b_{2}} p_{2}\left(x_{2}, t_{2}\right) F^{\prime}\left(t_{2}\right) d t_{2}=\int_{a_{2}}^{b_{2}} \int_{a_{1}}^{b_{1}} p_{2}\left(x_{2}, t_{2}\right) p_{1}\left(x_{1}, t_{1}\right) \frac{\partial^{2} f}{\partial t_{1} \partial t_{2}} d t_{1} d t_{2} \\
=\left(1-\gamma_{2}\right)\left(b_{2}-a_{2}\right) F\left(x_{2}\right)+\gamma_{2}\left[\left(b_{2}-x_{2}\right) F_{2}\left(b_{2}\right)+\left(x_{2}-a_{2}\right) F_{2}\left(a_{2}\right)\right] \\
\quad-\int_{a_{2}}^{b_{2}} F_{2}\left(t_{2}\right) d t_{2} \\
=\sum_{k=1}^{3} \sum_{j=1}^{3} C_{k 1} C_{j 2} f_{j k}-\sum_{j=1}^{3}\left(C_{j 1} I_{j 2}+C_{j 2} I_{j 1}\right)+\int_{a_{2}}^{b_{2}} \int_{a_{1}}^{b_{1}} f\left(t_{1}, t_{2}\right) d t_{1} d t_{2} .
\end{gathered}
$$

Assuming that both first partial derivatives of $f$ are bounded, we can 


\section{The Results}

simply write down the inequality

$$
\begin{aligned}
& \left|\int_{a_{2}}^{b_{2}} \int_{a_{1}}^{b_{1}} p_{2}\left(x_{2}, t_{2}\right) p_{1}\left(x_{1}, t_{1}\right) \frac{\partial^{2} f}{\partial t_{1} \partial t_{2}} d t_{1} d t_{2}\right| \\
& \quad \leq\left\|f_{t_{1}, t_{2}}^{\prime \prime}\right\|_{\infty}\left(\int_{a_{2}}^{b_{2}}\left|p_{2}\left(x_{2}, t_{2}\right)\right| d t_{2}\right)\left(\int_{a_{1}}^{b_{1}}\left|p_{1}\left(x_{1}, t_{1}\right)\right| d t_{1}\right) .
\end{aligned}
$$

Now, consider

$$
\begin{aligned}
G_{1}\left(x_{1}\right)= & \int_{a_{1}}^{b_{1}}\left|p_{1}\left(x_{1}, t_{1}\right)\right| d t_{1} \\
= & -\int_{a_{1}}^{\alpha_{1}}\left(t_{1}-\alpha_{1}\right) d t_{1}+\int_{\alpha_{1}}^{x_{1}}\left(t_{1}-\alpha_{1}\right) d t_{1} \\
& -\int_{x_{1}}^{\beta_{1}}\left(t_{1}-\beta_{1}\right) d t_{1}+\int_{\beta_{1}}^{b_{1}}\left(t_{1}-\beta_{1}\right) d t_{1} \\
= & \frac{1}{2}\left[\left(\alpha_{1}-a_{1}\right)^{2}+\left(x_{1}-\alpha_{1}\right)^{2}+\left(\beta_{1}-x_{1}\right)^{2}+\left(b_{1}-\beta_{1}\right)^{2}\right] \\
= & \frac{1}{2}\left[1+\left(2 \gamma_{1}-1\right)^{2}\right]\left[\left(\frac{b_{1}-a_{1}}{2}\right)^{2}+\left(x_{1}-\frac{a_{1}+b_{1}}{2}\right)^{2}\right] .
\end{aligned}
$$

Similarly, with $G_{2}\left(x_{2}\right)=\int_{a_{2}}^{b_{2}}\left|p_{2}\left(x_{2}, t_{2}\right)\right| d t_{2}$, we have

$$
G_{2}(x)=\frac{1}{2}\left[1+\left(2 \gamma_{2}-1\right)^{2}\right]\left[\left(\frac{b_{2}-a_{2}}{2}\right)^{2}+\left(x_{2}-\frac{a_{2}+b_{2}}{2}\right)^{2}\right] .
$$


Using (3), (4) and (5) and substituting (10), (12) and (13) into (11) will produce the result (2) and thus the theorem is proved.

The following result gives an Ostrowski type inequality for double integrals. It involves double and single integrals together with a function evaluation at an interior point.

Corollary 2 With the conditions as in Theorem 1, then

$$
\begin{gathered}
\mid\left(b_{1}-a_{1}\right)\left(b_{2}-a_{2}\right) f\left(x_{1}, x_{2}\right)-\left(b_{2}-a_{2}\right) \int_{a_{1}}^{b_{1}} f\left(t_{1}, x_{2}\right) d t_{1} \\
-\left(b_{1}-a_{1}\right) \int_{a_{2}}^{b_{2}} f\left(x_{1}, t_{2}\right) d t_{2}+\int_{a_{2}}^{b_{2}} \int_{a_{1}}^{b_{1}} f\left(t_{1}, t_{2}\right) d t_{1} d t_{2} \mid \\
\leq\left\|f_{t_{1}, t_{2}}^{\prime \prime}\right\|_{\infty}\left[\left(\frac{b_{1}-a_{1}}{2}\right)^{2}+\left(x_{1}-\frac{a_{1}+b_{1}}{2}\right)^{2}\right] \\
\times\left[\left(\frac{b_{2}-a_{2}}{2}\right)^{2}+\left(x_{2}-\frac{a_{2}+b_{2}}{2}\right)^{2}\right] .
\end{gathered}
$$

Proof: Place $\gamma_{1}=\gamma_{2}=0$ into equation (2).

Thus, the earlier results of [1] and [5, p.468] are reproduced as a special case of Theorem 1 . We note that unlike [1], the proof for Theorem 1 can be easily extended to more than two dimensions. 
Different values of the parameters $\gamma_{1}, \gamma_{2}, x_{1}$ and $x_{2}$ give rise to NewtonCotes type inequalities for functions with bounded derivatives. For example $\gamma_{1}=\gamma_{2}=0, x_{1}=\left(a_{1}+b_{1}\right) / 2$ and $x_{2}=\left(a_{2}+b_{2}\right) / 2$ produces the mid-point inequality; $\gamma_{1}=\gamma_{2}=1$ a trapezoid-like inequality and $\gamma_{1}=\gamma_{2}=1 / 3$ a Simpson's like inequality.

From Theorem 1 it is a simple matter to show that the tightest bound is obtained when $\gamma_{1}=\gamma_{2}=1 / 2$ and $x_{1}$ and $x_{2}$ are at their mid-points. That is for the average of the mid-point and trapezoid inequalities.

Remark 3 Let $f\left(t_{1}, t_{2}\right)=g\left(t_{1}\right) g\left(t_{2}\right)$ where $g:[a, b] \rightarrow \mathrm{R}$. If $g$ is differentiable and satisfies the condition that $\left\|g^{\prime}\right\|_{\infty}<\infty$, then, for $x_{1}=x_{2}=x$ and $\gamma_{1}=\gamma_{2}=\gamma$, we obtain a result from Theorem 1 which may be factored to recover the 3-point rule developed in [2], namely

$$
\begin{aligned}
\mid \int_{a}^{b} g(t) d t & -\gamma((x-a) g(a)+(b-x) g(b))-(1-\gamma)(b-a) g(x) \mid \\
\leq & \frac{\left\|g^{\prime}\right\|_{\infty}}{2}\left(1+(2 \gamma-1)^{2}\right)\left(\left(\frac{b-a}{2}\right)^{2}+\left(x-\frac{a+b}{2}\right)^{2}\right)
\end{aligned}
$$

In general, cubature formulae are written only in terms of function evaluations, but Theorem 1 approximates a double integral in terms of single integrals and function evaluations. Therefore we write down the following corollary which eliminates the one dimensional integrals by approximating 
them using the 3 -point rule in equation (15). The resulting inequality has a coarser bound than equation (2).

Corollary 4 Let $f$ be given as in Theorem 1. Then

$$
\begin{aligned}
&\left|\int_{a_{2}}^{b_{2}} \int_{a_{1}}^{b_{1}} f\left(t_{1}, t_{2}\right) d t_{1} d t_{2}-\sum_{k=1}^{3} \sum_{j=1}^{3} C_{k 1} C_{j 2} f_{j k}\right| \\
& \leq \frac{\left\|f_{t_{1}, t_{2}}^{\prime \prime}\right\|_{\infty}}{4}\left(1+\left(2 \gamma_{1}-1\right)^{2}\right)\left(1+\left(2 \gamma_{2}-1\right)^{2}\right) \\
& \quad \times\left[\left(\frac{b_{1}-a_{1}}{2}\right)^{2}+\left(x_{1}-\frac{a_{1}+b_{1}}{2}\right)^{2}\right]\left[\left(\frac{b_{2}-a_{2}}{2}\right)^{2}+\left(x_{2}-\frac{a_{2}+b_{2}}{2}\right)^{2}\right] \\
&+ \frac{1}{2}\left(1+\left(2 \gamma_{1}-1\right)^{2}\right)\left[\left(\frac{b_{1}-a_{1}}{2}\right)^{2}+\left(x_{1}-\frac{a_{1}+b_{1}}{2}\right)^{2}\right] \\
& \quad \times\left\{\gamma_{2}\left(x_{2}-a_{2}\right)\left\|f_{t_{1}, a_{2}}^{\prime}\right\|_{\infty}+\left(1-\gamma_{2}\right)\left(b_{2}-a_{2}\right)\left\|f_{t_{1}, x_{2}}^{\prime}\right\|_{\infty}\right. \\
&\left.\quad+\gamma_{2}\left(b_{2}-x_{2}\right)\left\|f_{t_{1}, b_{2}}^{\prime}\right\|_{\infty}\right\} \\
&+\frac{1}{2}\left(1+\left(2 \gamma_{2}-1\right)^{2}\right)\left[\left(\frac{b_{2}-a_{2}}{2}\right)^{2}+\left(x_{2}-\frac{a_{2}+b_{2}}{2}\right)^{2}\right] \\
& \times\left\{\gamma_{1}\left(x_{1}-a_{1}\right)\left\|f_{a_{1}, t_{2}}^{\prime}\right\|_{\infty}+\left(1-\gamma_{1}\right)\left(b_{1}-a_{1}\right)\left\|f_{x_{1}, t_{2}}^{\prime}\right\|_{\infty}\right. \\
&\left.\quad+\gamma_{1}\left(b_{1}-x_{1}\right)\left\|f_{b_{1}, t_{2}}^{\prime}\right\|_{\infty}\right\}
\end{aligned}
$$

Proof: Approximating each single integral in (2) by (15) and applying the triangle inequality produces the desired result. 
Remark 5 If $\gamma_{1}=\gamma_{2}=0$ and $x_{i}=\frac{a_{i}+b_{i}}{2}$, then

$$
\begin{gathered}
\left|\int_{a_{2}}^{b_{2}} \int_{a_{1}}^{b_{1}} f\left(t_{1}, t_{2}\right) d t_{1} d t_{2}-\left(b_{1}-a_{1}\right)\left(b_{2}-a_{2}\right) f\left(\frac{a_{1}+b_{1}}{2}, \frac{a_{2}+b_{2}}{2}\right)\right| \\
\leq \frac{\left\|f_{t_{1}, t_{2}}^{\prime \prime}\right\|_{\infty}}{16}\left(b_{1}-a_{1}\right)^{2}\left(b_{2}-a_{2}\right)^{2}+\frac{\left\|f_{t_{1}, \frac{a_{2}+b_{2}}{2}}^{\prime}\right\|_{\infty}}{4}\left(b_{2}-a_{2}\right)\left(b_{1}-a_{1}\right)^{2} \\
+\frac{\left\|f_{\frac{a_{1}+b_{1}}{2}, t_{2}}^{\prime}\right\|_{\infty}}{4}\left(b_{1}-a_{1}\right)\left(b_{2}-a_{2}\right)^{2} .
\end{gathered}
$$

\section{Numerical Results}

In this section the inequalities developed in Section 3 are used to approximate the double integral

$$
\int_{0}^{1} \int_{0}^{1} 1-e^{-x y} d x d y=0.203400400702947 .
$$

This integrand was chosen because integrating once in each direction is trivial. Namely, $\int_{0}^{1} 1-e^{-x y} d x=\frac{y+e^{-y}-1}{y}$ and $\int_{0}^{1} 1-e^{-x y} d y=\frac{x+e^{-x}-1}{x}$, but the double integral is not.

In Table 1, results are shown for the approximation to (18) using the rule and bound of (2). The numerical error is much smaller than the theoretical 
TABLE 1: The numerical and theoretical errors in computing (18) using (2) with $x_{1}=x_{2}=0.5$ and various values of $\gamma_{1}, \gamma_{2}$.

\begin{tabular}{|c|c||c|c|}
\hline$\gamma_{1}$ & $\gamma_{2}$ & Numerical Error & Theoretical Error \\
\hline \hline 0 & 0 & $1.5(-3)$ & $6.3(-2)$ \\
$\frac{1}{3}$ & $\frac{1}{3}$ & $5.4(-7)$ & $1.9(-2)$ \\
0.5 & 0.5 & $4.3(-4)$ & $1.6(-2)$ \\
1 & 1 & $6.5(-3)$ & $6.3(-2)$ \\
\hline
\end{tabular}

one and is smallest when Simpson's rule is applied $\left(\gamma_{1}=\gamma_{2}=\frac{1}{3}\right)$. The optimal theoretical bound is attained when $\gamma_{1}=\gamma_{2}=\frac{1}{2}$. It should be noted that $\gamma_{1}=\gamma_{2}=0$ approximates (18) with the "mid-point" rule and employs one function evaluation (at the midpoint of the region) and two one-dimensional integrals (along the bi-sectors). The "trapezoidal" rule uses four sample points (the boundary corners) and four one-dimensional integrals (along the boundary). All other values, that is $\gamma_{1}, \gamma_{2} \in(0,1)$, produces a rule that is a linear combination of the above and results in the use of nine sample points and six one-dimensional integrals.

To approximate (18) only in terms of function evaluations we use equation (16). The results are presented in Table 2. Since (16) is an approximation of (2), the results are qualitatively similar and quantitatively less accurate than those in Table 1 . Simpson's rule $\left(\gamma_{1}=\gamma_{2}=\frac{1}{3}\right.$, nine sample points) is more accurate than the midpoint rule $\left(\gamma_{1}=\gamma_{2}=0\right.$, one sample point) which in turn is more accurate than the trapezoidal rule $\left(\gamma_{1}=\gamma_{2}=1\right.$, 
TABLE 2: The numerical and theoretical errors in computing (18) using (16) with $x_{1}=x_{2}=0.5$ and various values of $\gamma_{1}, \gamma_{2}$.

\begin{tabular}{|c|c||c|c|}
\hline$\gamma_{1}$ & $\gamma_{2}$ & Numerical Error & Theoretical Error \\
\hline \hline 0 & 0 & $1.8(-2)$ & $4.6(-1)$ \\
$\frac{1}{3}$ & $\frac{1}{3}$ & $9.3(-4)$ & $2.2(-1)$ \\
0.5 & 0.5 & $1.0(-2)$ & $1.9(-1)$ \\
1 & 1 & $4.5(-2)$ & $3.8(-1)$ \\
\hline
\end{tabular}

four sample points). We note that the theoretical errors are symmetric about $\gamma_{1}=\gamma_{2}=\frac{1}{2}$ in Table 1 , but this is not the case in Table 2; these properties are easy to see by inspection of (2) and (16) respectively.

To illustrate the use of a cubature formula, we form a composite rule from the inequality (14).

Theorem 6 Let $f:\left[a_{1}, b_{1}\right] \times\left[a_{2}, b_{2}\right] \rightarrow \mathrm{R}$ bs as in Theorem 1. Let $I_{n}$ and $J_{m}$ be arbitrary divisions of $\left[a_{1}, b_{1}\right]$ and $\left[a_{2}, b_{2}\right]$, respectively, i.e.

$I_{n}: a_{1}=\xi_{0}<\xi_{1}<\cdots<\xi_{n}=b_{1}, \quad$ and $\quad J_{m}: a_{2}=\tau_{0}<\tau_{1}<\cdots<\tau_{m}=b_{2}$. 
Then we have the cubature formula

$$
\begin{aligned}
\int_{a_{2}}^{b_{2}} \int_{a_{1}}^{b_{1}} f\left(t_{1}, t_{2}\right) d t_{1} d t_{2} & =\sum_{i=0}^{n-1} \sum_{j=0}^{m-1} h_{i} \nu_{j} f\left(x_{i}, y_{i}\right)-\sum_{i=0}^{n-1} h_{i} \int_{a_{2}}^{b_{2}} f\left(x_{i}, t_{2}\right) d t_{2} \\
& -\sum_{j=0}^{m-1} \nu_{j} \int_{a_{1}}^{b_{1}} f\left(t_{1}, y_{j}\right) d t_{1}+R\left(f, I_{n}, J_{m}, \mathbf{x}, \mathbf{y}\right),
\end{aligned}
$$

where the remainder term $R$ satisfies the condition

$$
\begin{aligned}
& \left|R\left(f, I_{n}, J_{m}, \mathbf{x}, \mathbf{y}\right)\right| \leq\left\|f_{t_{1}, t_{2}}^{\prime \prime}\right\|_{\infty} \\
& \times \sum_{i=0}^{n-1} \sum_{j=0}^{m-1}\left[\left(\frac{h_{i}}{2}\right)^{2}+\left(x_{i}-\frac{\xi_{i}+\xi_{i+1}}{2}\right)^{2}\right]\left[\left(\frac{\nu_{j}}{2}\right)^{2}+\left(y_{j}-\frac{\tau_{j}+\tau_{j+1}}{2}\right)^{2}\right],
\end{aligned}
$$

and $h_{i}=\xi_{i+1}-\xi_{i}, \nu_{j}=\tau_{j+1}-\tau_{j}, x_{i} \in\left(\xi_{i}, \xi_{i+1}\right), y_{j} \in\left(\tau_{j}, \tau_{j+1}\right)$ for $i=$ $0,1, \ldots, n-1$ and $j=0,1, \ldots, m-1$.

Proof: Applying Corollary 2 on the interval $\left[\xi_{i}, \xi_{i+1}\right] \times\left[\tau_{j}, \tau_{j+1}\right]$, summing and using the triangle inequality produces the result. We omit the details.

Remark 7 If we were to use (19) to approximate the integral

$$
\int_{a_{2}}^{b_{2}} \int_{a_{1}}^{b_{1}} f\left(t_{1}, t_{2}\right) d t_{1} d t_{2}
$$


TABLE 3: The numerical and theoretical errors in evaluating (18) using the cubature rule in (19) for various values of $n, m$. Sampling occurs at the mid-point of each region.

\begin{tabular}{|c|c||c|c|c|}
\hline$n$ & $m$ & Numerical Error & Error ratio & Theoretical Error \\
\hline \hline 1 & 1 & $1.5(-3)$ & $\ldots$ & $6.3(-2)$ \\
2 & 2 & $1.0(-4)$ & 14.51 & $1.6(-2)$ \\
4 & 4 & $6.7(-6)$ & 15.61 & $3.9(-3)$ \\
8 & 8 & $4.2(-7)$ & 15.90 & $1.0(-3)$ \\
16 & 16 & $2.6(-8)$ & 15.98 & $2.0(-4)$ \\
32 & 32 & $1.6(-9)$ & 15.99 & $6.1(-5)$ \\
64 & 64 & $1.0(-10)$ & 16.00 & $1.5(-5)$ \\
128 & 128 & $6.6(-12)$ & 16.00 & $3.8(-6)$ \\
\hline
\end{tabular}

with a uniform grid and sampling at each mid-point, then the remainder $R$ is bounded by

$$
\left|R\left(f, I_{n}, J_{m}, \mathbf{x}, \mathbf{y}\right)\right| \leq\left\|f_{t_{1}, t_{2}}^{\prime \prime}\right\|_{\infty} \frac{\left(b_{1}-a_{1}\right)^{2}\left(b_{2}-a_{2}\right)^{2}}{16 n m}
$$

Table 3 shows the numerical and theoretical errors in applying the midpoint cubature rule (19) to evaluate the double integral (18) for an increasing number of intervals. The numerical error ratio suggests that this composite 
rule has convergence

$$
|R| \sim O\left(\frac{1}{n^{2} m^{2}}\right) .
$$

This contrasts with (21) which predicts a convergence rate of

$$
|R| \leq \frac{1}{16 n m}
$$

It should be noted that the development of the bounds in Section 3 assumes that the integrand is once differentiable. This condition admits a wider class of functions than the usual bounds for Newton-Cotes rules, but the error estimate will be more conservative if its applied, as it is here, to an integrand that is infinitely smooth. In addition, theoretical optimality occurs at $\gamma_{1}=\gamma_{2}=\frac{1}{2}$, while numerically this value seems to be $\gamma_{1}=\gamma_{2}=\frac{1}{3}$. Because of the behaviour of the integrand, Simpson's rule which is optimal (in the Newton-Cotes sense) for the class of fourth differentiable mappings, will be superior. The methods of Section 3 can be applied to smoother [3] as well as weighted mappings. Work is continuing in this direction. 


\section{References}

[1] N. S. Barnett and S. S. Dragomir. An Ostrowski type inequality for double integrals and application for cubature formulae. Soochow J. of Math, submitted, 1999. [ONLINE] Available online from the RGMIA: http://melba.vu.edu.au/ ${ }^{\text {rgmia/v1n1.html C674, C679, C679 }}$

[2] P. Cerone and S.S Dragomir. Three point quadrature rules involving, at most, a first derivative. SIAM J. Num. Anal., submitted, 1999. [ONLINE] Available online from the RGMIA: http://melba.vu.edu.au/rgmia/v2n4.html C673, C673, C673, C674, C680

[3] P. Cerone, S.S. Dragomir, and J. Roumeliotis. Some Ostrowski type inequalities for $n$-time differentiable mappings and applications.

Demonstratio Mathematica, accepted for publication, 1999. [ONLINE] Available online from the RGMIA: http://melba.vu.edu.au/ rgmia/v1n1.html C673, C687

[4] M. Golomb and H.F. Weinberger Optimal approximation and error bounds. In R.E. Langer, editor, On Numerical Approximation, pages 117-190, University of Wisconsin Press, Madison, 1959. C673

[5] D. S. Mitrinović, J. E. Pečarić, and A. M. Fink. Inequalities Involving Functions and Their Integrals and Derivatives. Dordrecht: Kluwer Academic, 1991. C679 
[6] J. Roumeliotis, P. Cerone, and S.S. Dragomir. An Ostrowski type inequality for weighted mappings with bounded second derivatives. $J$. KSIAM, accepted for publication, 1999. [ONLINE] Available online from the RGMIA:

http://melba.vu.edu.au/ ${ }^{\sim}$ rgmia/v1n1.html C673

[7] J.F. Traub and H. Woźniakowski. A General Theory of Optimal Algorithms. Academic Press, 1980.

C673 